\title{
Gestión del conocimiento: una perspectiva desde la gestión de la calidad y la gestión documental*
}

\section{Knowledge management: a perspective from quality management and document management}

Recibido: 27 de junio de 2013

Revisado: 26 de agosto de 2013 Aceptado: 09 de septiembre de 2013

María Janneth Álvarez Álvarez***

Universidad de La Salle

Martha Castro Rojas****

Icontec

Guillermo Peña Guarin ${ }^{* * * * *}$

Universidad Santo Tomás

\section{RESUMEN}

Gestionar el conocimiento que se produce durante el desarrollo de las actividades normales de una organización y lograr mejoras concretas son actividades que conducen a la obtención de ventajas competitivas. El sistema de gestión de la calidad basado en la norma
ISO 9001:2008 contiene disposiciones que se pueden equiparar con elementos para la gestión del conocimiento; sin embargo, este sistema no tiene referencias explícitas para la gestión documental, necesaria para la gestión del conocimiento, ya que tarde o temprano el conocimiento termina codificado en algún tipo de documento. Este estudio examina las convergencias

* Artículo de reflexión del grupo conformado para la investigación que se desarrolla entre la Universidad de La Salle, la Universidad Santo Tomás de Aquino y el Instituto Colombiano de Normas Técnicas (Icontec).

** Correspondencia: María Janneth Álvarez: Bibliotecóloga y archivista; magíster en Docencia; profesora e investigadora de la Universidad de La Salle, Bogotá (Colombia). Correo electrónico: mjalvarez@unisalle.edu.co

*** Martha Lucía Castro Rojas: Ingeniera química; especialista en Ingeniería Ambiental; jefe de Innovación y Cooperación de Icontec, Bogotá (Colombia).Correo electrónico: mcastro@icontec.org

**** Guillermo Peña Guarín: Ingeniero químico; magíster en Calidad y Gestión Integral; Profesor Investigador de la Universidad Santo Tomás, Bogotá (Colombia).Correo electrónico: guillermo.pena@usantotomas.edu.co. 
y complementariedades entre la gestión de la calidad según la norma ISO 9001:2008, la gestión del conocimiento de acuerdo con el informe UNE 412001:2008 y la gestión documental establecida en las norma ISO 30301:2011 y la guía ISO 15489-2:2001. Se identifican los aspectos relevantes que surgen de la comparación de estos tres sistemas de gestión y se proponen algunas directrices para su construcción simultánea en una organización.

Palabras clave: gestión del conocimiento, gestión documental, gestión de la calidad, ISO 9001.

\section{ABSTRACT}

Managing the knowledge that takes place during the development of the normal activities of an organization and achieving concrete improvements, leads to obtaining competitive advantages. The quality management system based on ISO 9001:2008 contains provisions that can be compared with elements of knowledge management, however it does not have explicit references to document management necessary for knowledge management since late or early knowledge ends codified in some kind of document. This study examines the convergences and complementarities between the quality management according to ISO 9001: 2008, knowledge management according to the report UNE 412001:2008 and records management established in the standard ISO 30301: 2011 and the guide ISO 15489- 2: 2001. Relevant issues arising from the comparison of these three management systems and some guidelines for simultaneous construction proposed in an organization were identified.

Keywords: Knowledge management, Management for records, Quality management, ISO 9001.

\section{INTRODUCCIÓN}

La nueva economía se estructura alrededor del manejo de la información y de su transformación en conocimiento, así como en su acelerada socialización gracias al uso intensivo, y cada vez más masivo, de las tecnologías de la información y la comunicación (TIC). Esto se confirma con el continuo desplazamiento de la cadena de valor hacia el campo de los servicios y del saber, en detrimento de la función tradicional basada en la manufactura y en los recursos tangibles ${ }^{1}$. Visto de esta manera, el conocimiento, su adquisición, manejo y conservación se han vuelto estratégicos y marcan diferencia entre las organizaciones.

Aunque todas las organizaciones usan y generan conocimiento, no todas lo hacen en forma consciente y ordenada, de manera que puedan extraer del flujo diario de actividades aquel conocimiento que es crítico para cumplir eficazmente su misión, transformarlo en códigos comprensibles para su uso interno, conservarlo para que esté prontamente disponible cuando se requiera y utilizarlo para la mejora continua de sus procesos y productos, así como para plantear las innovaciones que la encaminen al logro de su visión.

En este marco, este artículo pretende generar una reflexión acerca de cómo los sistemas de gestión de la calidad y de la documentación pueden ser considerados como precursores e impulsores organizacionales de la gestión del conocimiento, teniendo en cuenta, en el primer caso, su uso extendido en las organizaciones, y en el segundo, su valor en materia de organización y valorización de la información. Todo lo anterior se plantea bajo el supuesto de que la implementación de un sistema de gestión de la calidad no solo da fe del desempeño organizacional en esta materia, sino que

1 HANISCH Bastian, LINDER Frank, MUELLER Ana y WALD Andreas. En: Knowledge management in Project environments. Journal of Knowledge Management. 2009, vol. 13 n. ${ }^{\circ}$ 4. p. 148. 
también demuestra cómo la gestión documental es una práctica.

Aunque la gestión del conocimiento dentro de de las organizaciones no se ha materializado como un sistema de gestión normalizado y su desarrollo se maneja más como una teoría de administración, se considera que es posible identificar su correlación con los sistemas de gestión de la calidad y documental basados en la comparación de tres documentos de carácter normativo que describen individualmente los componentes y requisitos de implementación de cada uno de estos elementos de gestión: la norma "ISO 9001:2008. Sistemas de gestión de la calidad. Requisitos", la norma "ISO 30310:2011. Documentación e información. Sistema de gestión de documentos. Requisitos" (y una guía que la complementa, la ISO 15489-2. Gestión de documentos. Directrices) y el informe "UNE 412001:2008. Guía práctica de gestión del conocimiento”.

Igualmente, este análisis se basa en el supuesto de que los tres elementos de gestión tienen elementos comunes y complementarios los cuales, a su vez, pueden integrarse con otros sistemas y elementos de gestión.

Es importante reconocer que si bien los alcances de los sistemas de gestión en estudio y de la gestión del conocimiento son compatibles, tienen también elementos que no están interrelacionados y no se integran completamente. Este aspecto genera un reto para las organizaciones, el cual implica que en etapas tempranas de la instauración de los sistemas de gestión se identifiquen sus elementos comunes, con el fin de evitar que se dupliquen esfuerzos, se generen sinergias que los potencien en beneficio de todas las partes interesadas y se definan los elementos complementarios de dichos sistemas de gestión.

El propósito de este artículo es, por lo tanto, el de identificar las convergencias y complementariedades de los sistemas de gestión de la calidad y de la gestión de la documentación en relación con la gestión del conocimiento, como un paso inicial para establecer una ruta que ayude a su implementación conjunta.

\section{ANÁLISIS}

Para llevar a cabo este análisis, se describen inicialmente las características esenciales de los sistemas de gestión de la calidad, gestión documental y gestión del conocimiento a partir de los documentos normativos ISO 9001, ISO 30301 y UNE 412001, respectivamente. Posteriormente se efectúa un análisis de convergencias y complementariedades entre estos.

\section{Calidad}

Las organizaciones son sistemas sociales ${ }^{2}$ que adquieren su razón de ser al satisfacer necesidades específicas de una determinada población mediante la prestación de servicios y la entrega de productos; en compensación por estos productos y servicios, el cliente (usuario, beneficiario o el Estado) retribuye económicamente a la organización para que esta logre sus objetivos.

Por otra parte, a la medida o al grado en que los productos y servicios que una organización le entrega a sus clientes satisfacen sus expectativas, se le denomina "la calidad". En consecuencia, se puede hablar de "buena calidad", "excelente calidad", "regular calidad" 0 incluso de una "pésima calidad", según ese grado de satisfacción.

Para lograr una adecuada calidad es necesario planificar las actividades, llevar a cabo esos planes y controlarlos, es decir, hay que gestionar la calidad. La gestión de la calidad tiene por finalidad garantizar que las expectativas (requisitos) de los clientes se entiendan

2 ACKOFF, Russell L. Systems thinking and thinking systems. En: System Dynamics Review, 1994, vol. 10, n. ${ }^{0} 2-3$, p. 175-188. 
y que además se cumplan de manera sostenida en el tiempo, articulando los múltiples factores organizacionales que se entrelazan en los procesos, para contribuir así a la sostenibilidad organizacional. Dicho de otro modo, la gestión de la calidad se encarga de asegurar "la calidad".

Si bien la calidad es inherente a la actividad humana dado que se satisface una necesidad o expectativa, la concepción moderna de la gestión de la calidad surge con la Revolución Industrial, con "el empuje de las necesidades de estandarización e intercambiabilidad que crea el modelo de producción en serie"3 y ha evolucionado con el impulso de algunos líderes pioneros (gurús de la calidad), que han creado sus propias "escuelas". Algunos de los líderes más representativos han sido:

Walter Andrew Shewhart. Fue el primero en proponer el control estadístico de la calidad y el uso de gráficos de control de productos manufacturados. Es el creador del ciclo PHVA, el cual es una estrategia de mejora continua de la calidad en cuatro pasos: planificar el proceso, hacerlo o ejecutarlo según lo planificado, verificar los avances y logros, y actuar de acuerdo con los resultados de la verificación. A este ciclo de mejora también se le conoce como el "ciclo de la gestión", y es uno de los conceptos fundamentales de la gestión de la calidad.

W. Edwards Deming. Apoyó el proceso de reconstrucción de Japón luego de la Segunda Guerra Mundial, impulsando en ese país las teorías aprendidas de Walter A. Shewhart relacionadas con el ciclo PHVA e integrando los catorce puntos de su teoría, que son principios fundamentales para la gestión y transformación de la eficacia empresarial. Según este enfoque, la gerencia de la organización juega un papel de liderazgo

3 CAMISÓN, César; CRUZ, Sonia y GONZÁLEZ, Tomás. Gestión de la calidad: conceptos, enfoques modelo y sistemas. Madrid. Pearson Educación, 2007. p. 78. y participa en la generación de cambios culturales en toda la organización: "Deming exige un cambio fundamental en el concepto que de la organización tienen las personas que la administran", siendo este aspecto esencial para su enfoque de la gestión de la calidad.

Joseph M. Juran. Para Juran, la calidad es la conformidad con las expectativas del cliente y lograr utilidades para la organización en la medida que se disminuya 0 minimice el costo de la no calidad. Enfatiza en la importancia que los administradores y los empleados tienen en el logro de una buena calidad, y considera la organización como un sistema: "la gestión de la calidad por toda la empresa (GCTE) es un enfoque sistémico para establecer y cumplir los objetivos de la calidad"5. Juran aporta al mejoramiento continuo desde lo que denomina la "trilogía de la calidad": la planeación de la calidad, el control sobre las operaciones y la mejora continua.

Philip Bayard Crosby. Para Crosby, la calidad se define en términos de la conformidad con las especificaciones del cliente, es decir, que la medida de la calidad es el valor del incumplimiento y que la política debe ser la de "cero defectos". Considera que todo trabajo es un proceso en sí mismo y que, por lo tanto, no puede ser tomado como algo aislado, sino como parte de la cadena con que se interrelaciona. Para Crosby, la gestión de la calidad se sustenta en la prevención y en lograr trabajar sin errores, porque los costos de la no calidad son más grandes para la organización que los costos de la prevención.

Kaoru Ishikawa. Lideró la gestión de la calidad en el Japón con un método basado en el enfoque a toda la

4 GITLOW, Howards. GITLOW, Shelly. Cómo mejorar la calidad y la productividad con el método Deming. Bogotá: Grupo Editorial Norma, 1991. p. 12.

5 JURAN, Joseph. Juran y la planificación para la calidad. Madrid. Díaz de Santos Ediciones, 1990. p. 217. 
organización. Según Ishikawa, la gestión de la calidad es "una revolución conceptual en la gerencia", lo cual implica la participación desde la alta dirección hasta los niveles más bajos. Su enfoque se aplica no solo a los bienes, sino también a los servicios y se centra en desarrollar, diseñar y elaborar productos que sean económicos, útiles y siempre satisfactorios para el consumidor. En la escuela de Ishikawa el cliente es considerado como parte del proceso, denominándolo como cliente interno, siendo el usuario final el cliente externo.

Gen'Ichi Taguchi. Aborda la gestión de la calidad desde dos perspectivas: la primera se relaciona con la construcción de la calidad desde la misma fase de diseño de procesos o de productos; la segunda se refiere al uso de métodos y técnicas estadísticas. La preocupación de Taguchi es la pérdida de confianza del cliente; al respecto, plantea la denominada "función de pérdida" como un recurso estadístico utilizado para la calidad, ya que el costo de la no calidad lo siente la sociedad en su totalidad. Aunque "la metodología de Taguchi ha despertado controversia" ${ }^{7}$, lo importante de este autor radica en la necesidad de contar con un diseño robusto y a partir de este determinar los problemas de calidad para no tener que resolverlos en la fase de producción.

El pensamiento de estos autores, junto con el de otros más, ha delineado el actual concepto de gestión de la calidad. En palabras de Camisón et al:: "la gestión de la calidad se ha construido históricamente como una compleja combinación de ideología y métodos que se han aplicado en la praxis (...) para afrontar problemas complejos de dirección de organizaciones". Añaden

6 ISHIKAWA, Kaoru. ¿Qué es el control total de la calidad? Bogotá. Editorial Noma, 1986. p. 98.

7 JURAN, J.M. y GRYNA, Frank. Análisis y planeación de la calidad. México. McGraw-Hill, 1993. p. 260.

8 ISHIKAWA, Kaoru. ¿Qué es el control total de la calidad? Bogotá. Editorial Noma, 1986. p. 98. que la gestión de la calidad es "una función de la dirección que se agrega a otras existentes anteriormente, como la gestión comercial"; es decir, que la gestión de la calidad adquiere una connotación de carácter estratégico dentro de la organización.

En el año 1987, la International Organization for Standardization (ISO) publicó un conjunto de normas técnicas internacionales a las que denominó la serie ISO 9000, las cuales fueron actualizadas posteriormente en los años 1994, 2000 y 2008. En la última versión, el enfoque principal de las normas es el de considerar a las organizaciones como un gran sistema, y a la gestión de la calidad como uno de los subsistemas que la conforman. Este enfoque sistémico de la organización consolidó los esfuerzos que en este campo desarrollaron los pioneros de la calidad antes mencionados, los cuales se plasmaron en los ocho principios de la gestión de la calidad que reflejan las mejores prácticas para dirigir una organización hacia la mejora de su desempeño frente a los clientes. Estas normas son genéricas, es decir, universales y aplicables a cualquier organización sin importar su naturaleza, tamaño o propósito.

La serie ISO 9000 enfatiza en la gestión por procesos y establece que la organización debe determinar los procesos, su secuencia e interacciones, los criterios y métodos para operarlos y controlarlos, además de implementar las acciones necesarias para su mejora continua ${ }^{10}$.

Es importante destacar que estas normas técnicas se definen como normas de sistemas de gestión de la calidad, entendiendo como sistema de gestión a un "sistema para establecer la política y los objetivos, y para

9 Ibíd., p. 56.

10 INSTITUTO COLOMBIANO DE NORMAS TÉCNICAS Y CERTIFICACIÓN. Sistemas de gestión de la calidad. Requisitos. NTC-ISO 9001:2008. Bogotá D.C.: El Instituto, 2009. 35 p. 
lograr dichos objetivos" $"$. En otras palabras, el sistema de gestión de la calidad debe establecer la política y los objetivos de la calidad, además de organizar los procesos, de manera que se logren los objetivos de la calidad establecidos; por lo tanto, la formulación de los objetivos es vital y, en consecuencia, estos deben estar conectados directamente con la estrategia organizacional.

La norma "NTC-ISO 9001:2000. Sistemas de gestión de la calidad. Requisitos", tiene como elementos destacables los siguientes:

- Responsabilidad de la dirección. Implica las actividades que la alta dirección de la organización debe adelantar, por ejemplo, el enfoque hacia el cliente para la definición de la política y los objetivos de la calidad, la planificación del sistema, la asignación de responsabilidad y autoridad, los procesos de comunicación entre las personas y una revisión general periódica para identificar oportunidades de mejora.

- Gestión de los recursos. Asociada tanto a la asignación de los recursos físicos y económicos como a los recursos humanos necesarios para alcanzar los objetivos establecidos. En este punto resultan esenciales los requerimientos para contar con personas competentes, con base en su educación, formación, habilidades y capacitación; también hay una exigencia por lograr la concienciación de las personas en cuanto a su papel dentro del sistema de gestión de la calidad.

- Los procesos para la realización del producto. La norma detalla todos los aspectos que influyen para la planificación y el control de los procesos operativos, es decir, aquellos que permiten la

11 INSTITUTO COLOMBIANO DE NORMAS TÉCNICAS Y CERTIFICACIÓN. Sistemas de gestión de la calidad. Fundamentos y vocabulario. NTC-ISO 9000:2005. Bogotá D.C.: El Instituto, 2005, p. 10. elaboración de un producto o la prestación de un servicio. Incluye "la trazabilidad", esto es, la recolección durante las actividades operativas de los datos que se consideren necesarios para el posterior análisis de los procesos y de los productos, y que permitan generar la información necesaria para encontrar sus fortalezas y debilidades.

- Medición, análisis y mejora. Busca el aprendizaje organizacional a partir de la solución de problemas mediante la toma de acciones correctivas y de utilizar la información recolectada para la toma de acciones preventivas que impidan la presencia de productos, servicios o procesos que incumplan los parámetros planificados.

- Requisitos de la documentación. La norma plantea la necesidad de elaborar y controlar, en la extensión que la misma organización considere prudente, dos tipos de documentos: aquellos que indican "cómo se debe proceder", tales como procedimientos, instructivos o manuales, y aquellos que evidencien "cómo se procedió", es decir, los registros de las actividades.

Para la teoría del sistema de gestión de la calidad, la satisfacción de las necesidades implica la puesta en práctica de determinadas formas de proceder, de acciones articuladas entre las diferentes personas que conforman la organización, de "haceres" y de "no haceres" que en su conjunto constituyen un conocimiento particular y propio de cada organización. Se podría afirmar que la manufactura de bienes y la prestación de servicios por parte de una organización son una manifestación materializada del conocimiento con la que esta cuenta y que debería gestionar adecuadamente para obtener el máximo beneficio de dicho conocimiento.

Desde esta misma perspectiva, los sistemas de gestión de la calidad están orientados a proporcionar un marco de operación claro y eficaz encaminado a lograr 
la satisfacción de los clientes. La construcción de este marco, tal y como lo plantea Sara Artiles ${ }^{12}$, tiene sustento en la gestión documental en la medida en que se debe describir de manera objetiva lo que "se sabe hacer bien”, permitiendo conservar y transmitir el conocimiento organizacional.

Lo anterior hace evidentes las relaciones que existen entre la calidad, la documentación y el conocimiento, pero ¿cómo llevar a la práctica esa relación? Para aproximarnos a una respuesta, es necesario mirar de cerca la gestión documental y la gestión del conocimiento.

\section{La gestión documental}

La presión de las tecnologías de la información (TIC) no solo ha influenciado la búsqueda de nuevas orientaciones para el manejo de novedosos soportes documentales, sino que ha incidido sobre la gestión de los documentos. Para entender la importancia de la documentación en una organización y la necesidad de gestionar adecuadamente dichos documentos, podemos remitirnos a la guía técnica GTC-ISO/TR 10013, la cual plantea las razones y los beneficios de tener documentado un sistema de gestión de la calidad, dentro de los que se destacan los siguientes: ${ }^{13}$

- Permite y facilita la comunicación del sistema de gestión a todas las partes interesadas (proveedores, empleados, clientes, gobierno, accionistas, etc.)

- Suministra un marco de operación claro y eficiente, pues permite declarar la forma en que las actividades se llevarán a cabo.

12 ARTILES, Sara. La gestión documental, de información y el conocimiento en la empresa. En: ACIMED. 2009, vol. 19, n. ${ }^{\circ}$ 5, 37 p.

13 INSTITUTO COLOMBIANO DE NORMAS TÉCNICAS Y CERTIFICACIÓN. Directrices para la documentación del sistema de gestión de la calidad. GTC-ISO/TR 10013. Bogotá, El Instituto, 2002. p. 5.
- Provee evidencia objetiva de cómo se alcanzan los resultados y genera una base para evaluar y mejorar la eficacia y adecuación continua del sistema de gestión de la calidad.

- Es la base para la formación inicial y la actualización periódica de los empleados.

Adicionalmente, en Colombia la Ley General de Archivos regula la función archivística del Estado en cuanto a la administración pública en sus diferentes niveles, y en aquellas entidades privadas que cumplen funciones públicas. El sistema de gestión para los documentos se define como un "conjunto de actividades administrativas y técnicas, que se realizan para el manejo y organización de la documentación que se produce y recibe por la entidad en el ejercicio de sus funciones" ${ }^{14}$, Lograr el aseguramiento de este recurso en una entidad permite demostrar la existencia de un proceso de gestión y cumplimiento de estándares, indicadores de que la entidad ha establecido un sistema de gestión de documentos para los procesos, los procedimientos y las evidencias que se generan en su interior.

La gestión de los documentos se relaciona con el diseño normalizado de los documentos, el control tanto de la duplicidad como de versiones; la simplificación, el control y uso de procedimientos; la adecuada organización e implementación de los procesos archivísticos de clasificación, ordenación y descripción de la documentación de una organización; la conservación, valoración y disponibilidad para la toma de decisiones y la eliminación de documentos que carecen de valor para la gestión.

Los denominados "sistemas de gestión" han tenido gran significación en todos los campos y disciplinas,

14 Colombia. Ley 594 de 2000. Por medio de la cual se dicta la Ley General de Archivos y se dictan otras disposiciones. Bogotá D.C., 14 de julio de 2000. 
y la gestión documental no ha sido la excepción. En 1996, los archivistas australianos presentaron la primera versión de una norma, denominada AS4390, la cual inició el camino para la presentación, implementación e implantación de normas de calidad para los procesos archivísticos, aspecto que revolucionó el contexto de los documentos de archivo y de la gestión documental en general. La norma propone nuevas y mejores prácticas para los procesos de la gestión de documentos, como su captura, registro, clasificación y conservación, entre otros.

Posteriormente se generaron otras normas técnicas para la gestión de los documentos y la información, como la norma "ISO 23081-1:2006. Procesos de gestión de documentos. Metadatos para la gestión de documentos" y la norma "ISO 22310:2006. Información y documentación. Directrices para los redactores de normas". Sin embargo, son dos las normas que marcan un hito en la gestión documental. La primera de ellas surgió en el año 2001 y se denominó "ISO 15489-1. Información y documentación. Gestión de documentos", que explicita las orientaciones generales para la autenticidad, fiabilidad, integridad y disponibilidad de los documentos que una organización produce 0 recibe. Esta norma establece que la gestión de documentos trae beneficios como los de poder "realizar las actividades en una forma ordenada, eficaz y responsable; prestar servicios de un modo coherente y justo; respaldar y documentar la toma de decisiones; garantizar la continuidad en caso de catástrofe y mantener una memoria corporativa, personal o colectiva" ${ }^{15}$, entre otros.

La segunda norma técnica clave surgió en 2011. Se trata de la ISO 30301 "Información y documentación. Sistema de gestión documental. Requisitos",

15 INTERNATIONAL ORGANIZATION FOR STANDARDIZATION. Information and Documentation - Records Management -Part 1. ISO 15489-1:2001. Geneva: Same, 2001. p. 6. la cual se enfoca en los requisitos mínimos que un sistema de gestión documental debe cumplir; está dispuesta según la "estructura de alto nivel" que promueve la ISO para unificar la distribución de los requisitos de un sistema de gestión y es, por supuesto certificable, es decir, un organismo certificador de tercera parte puede dar fe de que estos requisitos se cumplen de forma sistemática en una organización. Esta norma es aplicable por toda organización que desee "establecer, implementar, mantener y mejorar un sistema de gestión documental para apoyar su negocio" ${ }^{16}$ y propone que se establezcan los procesos necesarios para la gestión documental, incluyendo los de su creación y control.

La gestión de los documentos constituye, desde esta óptica, una práctica archivística que incluye la fijación de políticas y normas, la asignación de responsabilidades, el establecimiento de procesos y procedimientos para que un documento sirva de soporte auténtico, con fiabilidad, integridad y de conformidad con las actividades propias de la entidad, que garantice la continuidad de las actividades, además del cumplimiento de las normas legales.

La norma técnica ISO 30301 hace referencia a esta integralidad en lo relacionado con procesos y controles para los documentos desde su creación, cuyo requisito fundamental consiste en que los documentos permanezcan accesibles en el tiempo que sea necesario. La norma es muy clara en este sentido cuando requiere de la implementación de una política y unos objetivos de gestión documental, así como de la definición de controles desde el origen del documento hasta su destino final, con el fin de facilitar su consulta, conservación y utilización para la toma de decisiones.

16 INTERNATIONAL ORGANIZATION FOR STANDARDIZATION. Information and Documentation - Management System for records. ISO 30300:2011. Geneva: Same, 2011. p. 1. 
El capítulo 8 de la norma técnica ISO 30301, titulado "Operación", hace referencia a la planeación y el control operacional de la gestión documental, así como al diseño de los procesos para los registros y a su implementación. Las directrices que la norma técnica ISO 15489-2 plantea brindan la orientación requerida para llevar a cabo estas actividades y, en consecuencia, permiten apreciar la forma como estas dos normas técnicas se articulan. Hay otros aspectos de la norma técnica ISO 15489-2 que convergen en la norma ISO 30301 y que esta última incorpora por tratarse de una norma técnica sobre el sistema de gestión documental, que referencia los aspectos operacionales de la gestión de los documentos.

Por otra parte, la norma ISO/TR 10013:2001 establece que "la organización de la documentación del sistema de gestión de la calidad normalmente sigue a los procesos de la organización..." ${ }^{17}$, señalando que los procesos identificados por la propia organización en su sistema de gestión de la calidad son el hilo conductor para la gestión de la documentación. El valor que aporta la gestión de la calidad a las organizaciones, desde el punto de vista documental, radica en identificar y documentar de manera objetiva lo que "se sabe hacer bien", es decir, el conocimiento organizacional, pero, además, en el énfasis que hace en la necesidad de dejar rastro de lo que se hace, esto mediante registros, bases de datos y publicaciones.

La gestión de los documentos es parte integral de las actividades de las organizaciones y se dirige hacia la eficiencia, la eficacia y la preservación de la memoria institucional. Sin embargo, se presenta una dificultad debido a la falta de interacción entre la gestión de la calidad y la gestión documental, que generalmente son vistas como sistemas independientes, lo cual no

17 INTERNATIONAL ORGANIZATION FOR STANDARDIZATION. Guidelines for quality management system documentation. ISO/TR 10013:2001. Geneva: Same, 2001. 14 p. solo incide negativamente sobre el desempeño organizacional, sino que impide la capitalización del conocimiento que se genera y limita la formulación de propuestas innovadoras.

\section{La gestión del conocimiento}

En el cambiante mundo en el que se desenvuelven las organizaciones, la habilidad para "aprender a aprender" se convierte en una ventaja competitiva. Pero las organizaciones solo aprenden a través de personas que aprenden, por lo cual cabe preguntarse: "¿porqué una persona debe compartir lo que sabe? ¿Qué motivos le llevan a realizar un esfuerzo adicional a su trabajo cotidiano? ¿Para qué?" ${ }^{18}$. Una forma de resolver esta encrucijada es brindando las condiciones para que las personas se sientan motivadas y comprometidas, lo cual se puede lograr mediante dos estrategias principales: en primer lugar, crear una cultura organizacional que promueva el aprendizaje continuo y valore el hecho de compartir el conocimiento; en segundo lugar, crear los mecanismos concretos que faciliten que ese conocimiento individual se haga explícito y se transforme en conocimiento organizacional.

Dado que el conocimiento es fruto del aprendizaje individual, es necesario que pase de los actores individuales a los grupos, y de estos a la organización en su conjunto, para lo cual se requiere "gestionarlo". En el marco del informe UNE 412001:2008, la gestión del conocimiento se define como "la dirección planificada y continua de procesos y actividades para potenciar el conocimiento e incrementar la competitividad a través del mejor uso y creación de recursos de conocimiento individual y colectivo" ${ }^{19}$. Es decir, que la gestión del

18 ASOCIACIÓN ESPAÑOLA DE NORMALIZACIÓN Y CERTIFICACIÓN. Guía práctica de gestión del conocimiento. UNE 412001:2008. Madrid: La Asociación, 2008. p. 35

19 Ibíd., p.8. 
conocimiento implica tanto la creación de la cultura organizacional apropiada como el desarrollo de los mecanismos para hacer que el conocimiento fluya a través de la organización. Para que ese conocimiento realmente circule, se deben considerar los procesos que la conforman. Una vez definidos estos procesos, pueden establecerse los conocimientos que se requieren para llevarlos a cabo, es decir, para planificarlos, ejecutarlos, controlarlos y mejorarlos. A la vez, en la medida en que los procesos se ejecutan y se controlan, surgen los registros con datos valiosos que posteriormente pueden transformarse en nuevo conocimiento.

Los datos se entienden como la representación de un hecho de la realidad; esta representación puede ser análoga o digital. Cuando los datos se analizan y adquieren significado se convierten en información; el análisis de la información nos lleva al conocimiento, es decir, al "saber". Ese saber se debe transformar en competencias individuales, esto es, en "saber hacer”, para que realmente se genere un aprendizaje organizacional.

La gestión del conocimiento ha evolucionado en las organizaciones, pasando de ser un recurso para el almacenamiento y la recuperación de datos, a un método para facilitar el flujo de la información y, finalmente, a un sistema para crear valor alineado con la estrategia de la organización.

La gestión del conocimiento implica los siguientes procesos: creación, identificación, captura, adaptación, organización, almacenamiento y aplicación del conocimiento. Es mediante estos procesos que se establece la circulación del conocimiento en la organización ${ }^{20}$. La etapa de aplicación del conocimiento realimenta a la de creación del conocimiento, con lo cual se genera un ciclo cibernético que conduce a la mejora continua.

20 Ibíd., p.
Estos procesos para la gestión del conocimiento consisten en:

- Creación del conocimiento. Este proceso se orienta a identificar y definir las estrategias explícitas que la organización ha establecido como indispensables para desarrollar conocimiento nuevo 0 para adquirirlo.

- Identificación del conocimiento. Consiste en determinar cuáles son los conocimientos que la organización requiere para funcionar y dar cumplimiento a su misión, y cuáles son necesarios para proyectarse en el logro de su visión.

- Captura del conocimiento. Este proceso hace referencia a identificar en cuáles etapas y en qué forma se captan los datos y la información necesarios para generar el conocimiento.

- Adaptación del conocimiento. Este proceso determina cómo los datos y la información se procesan, se analizan y se adaptan a las necesidades organizacionales, para transformarlos en conocimiento útil.

- Organización del conocimiento. Incluye la codificación y documentación del conocimiento en los medios adecuados y su clasificación según unas categorías definidas, de forma que se facilite su posterior recuperación.

- Almacenamiento del conocimiento. Proceso relacionado con los mecanismos utilizados para conservar la integridad y asegurar la accesibilidad del conocimiento, sin importar el medio en que este se encuentre, durante los plazos previstos para la conservación.

- Aplicación del conocimiento. Proceso que inicia con la capacitación de las personas en el nuevo conocimiento y su uso en la cotidianeidad de las 
actividades, generando mejoras en el desempeño organizacional.

Pero, además, el documento UNE 412001 es enfático al establecer que la cultura organizacional es el factor más importante para manejar con éxito el conocimiento, lograr una participación activa de los miembros de la organización y organizar la puesta en marcha del sistema de gestión del conocimiento. En otras palabras, el primer paso consiste en crear la cultura adecuada para la gestión del conocimiento.

La gestión documental se define como la "forma de analizar y facilitar la explotación de la información contenida en los documentos existentes en la organización y que soportan sus operaciones"21. Como la gestión documental se rige fundamentalmente por los principios derivados de la teoría de sistemas y del ciclo de vida de la información ${ }^{22}$, resulta plenamente compatible con la gestión del conocimiento, pues brinda los recursos especializados para administrar los documentos, de cualquier tipo, en los cuáles, tarde o temprano, todo el conocimiento explícito de una organización queda plasmado.

Buena parte de ese conocimiento proviene del quehacer diario de la organización en la ejecución de los procesos que permiten satisfacer las necesidades de los clientes y hace referencia a las capacidades específicas que se han desarrollado para mejorar las relaciones actuales y futuras con los clientes; dicho de otro modo, el conocimiento plasmado en los documentos proviene de la gestión de la calidad a la vez que la realimenta.

21 Ibíd., p.8

22 ARTILES. Op. cit., p. 10.

\section{Comparación}

Conocidas las características, ventajas y limitaciones de la gestión de la calidad, la documental y la del conocimiento, vistas cada una como un sistema independiente, surge la cuestión de cómo articular estos sistemas de gestión para que actúen como uno solo, de manera que se refuercen sus beneficios, se eliminen las redundancias y se genere una sinergia en la que tanto la organización como sus clientes resulten favorecidos.

En primer lugar, hay que dilucidar si uno de estos tres sistemas de gestión tiene mayor importancia o si alguno de ellos contiene al otro. Según el pensamiento sistémico, para entender un sistema abierto conviene hacer una observación de este como un todo, es decir, establecer cómo se relaciona ese sistema con los demás y con su entorno; por ello se deben entender "las interacciones" del sistema en estudio con los demás.

Por una parte, la gestión de la calidad tiene como propósito garantizar que los acuerdos con los clientes se cumplan correctamente y de manera continua; ello implica un entendimiento sobre cuáles son las necesidades de esos clientes y de cómo se debe proceder internamente para darles cumplimiento, es decir, se requiere un conocimiento previo. Ahora bien, para que ese conocimiento se pueda transmitir, minimizando la posibilidad de errores, debería estar documentado, registrado y actualizado. La gestión del conocimiento facilitaría la circulación del conocimiento requerido para satisfacer las expectativas de los clientes, en tanto que la gestión documental aseguraría el debido almacenamiento y la adecuada protección de los documentos para que estén siempre disponibles.

Adicionalmente, se produce nuevo conocimiento durante el desarrollo de las actividades que una organización ha establecido para elaborar sus productos y prestar los servicios. Ese conocimiento puede estar 
como un dato registrado, que mediante un posterior análisis se convierte en información, o también como un conocimiento a nivel individual, fruto de la experiencia. Canalizar ese conocimiento para no perderlo, y convertirlo luego en conocimiento colectivo sería una función de la gestión del conocimiento. Entonces, el sistema de gestión de la calidad requiere de conocimiento previo para su correcta operación, pero, a la vez, genera un conocimiento adicional cuando opera.

Pero no es posible hacer una adecuada gestión del conocimiento si este conocimiento no tiene un soporte que elimine las posibles ambigüedades en su interpretación, es decir, si no está documentado. El conocimiento es información cierta y pertinente que ha surgido bien sea del análisis de los datos recolectados, o bien extractado de la experiencia de los individuos, y que puede ser de alguna forma aprovechado.

Un análisis comparativo nos permite comprender las conexiones entre la gestión del conocimiento, la documental y la de la calidad.

En la tabla 1 se observa la comparación de los procesos de la gestión del conocimiento que plantea el informe UNE 412001:2008 con los modelos de gestión documental ISO 30301:2011 (e ISO 154892:2001) y el sistema de gestión de la calidad basado en ISO 9001:2008, para identificar sus convergencias y complementariedades.

Como criterios para llevar a cabo la comparación se decide tomar como eje articulador a los procesos de la gestión del conocimiento más el principio de que la cultura organizacional es clave para la gestión del conocimiento. Así mismo, se decide que un requisito o lineamiento de las normas de gestión de la calidad y de la documental solo se asocia a un proceso de la gestión del conocimiento, a aquel con el cual tenga una relación directa.

El resultado de la comparación muestra que hay requisitos de ISO 9001 que convergen o se relacionan para todos los procesos de la gestión del conocimiento.

Por otra parte, la comparación general de la norma UNE 412001 con los documentos sobre gestión de la documentación ISO 30301 e ISO 15489-2 muestra convergencia para seis de los procesos de la gestión del conocimiento, excepto para los de creación del conocimiento y adaptación del conocimiento. 
Tabla 1. Relación entre los procesos de gestión del conocimiento, el sistema de gestión de la calidad y el sistema de gestión documental

\begin{tabular}{|c|c|c|}
\hline $\begin{array}{l}\text { Procesos de gestión } \\
\text { del conocimiento } \\
\text { (según UNE 412001:2008) }\end{array}$ & $\begin{array}{l}\text { Gestión de la calidad } \\
\text { (según ISO 9001:2008) }\end{array}$ & $\begin{array}{l}\text { Gestión documental } \\
\text { (según ISO 30301:2011 - } \\
\text { ISO 15489-2:2001) }\end{array}$ \\
\hline $\begin{array}{l}\text { 1. Creación de la cultura } \\
\text { orǵanizacional necesaria para } \\
\text { gestionar el conocimiento }\end{array}$ & $\begin{array}{l}\text { 5.5.1 “...asegurarse de que las responsabilidades } \\
\text { y autoridades están definidas y son comunicadas } \\
\text { dentro de la organización”. } \\
\text { 5.5.2. c “...asegurarse de que se promueva la toma } \\
\text { de conciencia de los requisitos del cliente en todos } \\
\text { los niveles de la organización”. } \\
\text { 5.5.3 “....asegurarse de que se establecen los } \\
\text { procesos de comunicación apropiados dentro de la } \\
\text { organización”. } \\
\text { 6.2.2.d "....asegurarse de que su personal es } \\
\text { consciente de la pertinencia e importancia de sus } \\
\text { actividades y de cómo contribuyen al logro de los } \\
\text { objetivos de la calidad”. }\end{array}$ & 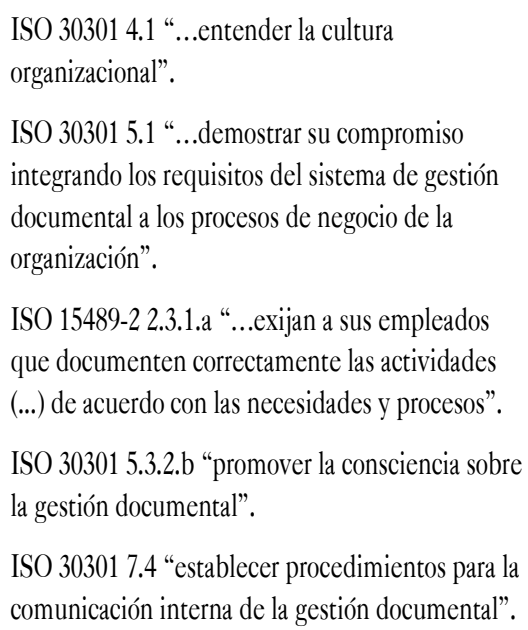 \\
\hline $\begin{array}{l}\text { 2. Creación del conocimiento } \\
\text { Estrategias explícitas para } \\
\text { desarrollar conocimiento o } \\
\text { adquirirlo }\end{array}$ & $\begin{array}{l}\text { 7.3.1 "...planificar y controlar el diseño y desarrollo } \\
\text { del producto". } \\
\text { 8.5.2 "...tomar acciones para eliminar las causas de } \\
\text { las no conformidades con objeto de prevenir que } \\
\text { vuelvan a ocurrir". } \\
\text { 8.5.3 “...tomar acciones para eliminar las causas de } \\
\text { las no conformidades potenciales para prevenir su } \\
\text { ocurrencia”. }\end{array}$ & \\
\hline $\begin{array}{c}\text { 3. Identificación del } \\
\text { conocimiento } \\
\text { Qué conocimientos se requieren } \\
\text { para apoyar la estrategia }\end{array}$ & $\begin{array}{l}\text { 5.4.2.a "....asegurarse de que la planificación del } \\
\text { sistema de gestión de la calidad se realiza, con el } \\
\text { fin de cumplir los requisitos". } \\
\text { 7.1"... planificar y desarrollar los procesos } \\
\text { necesarios para la realización de producto". } \\
\text { 7.2.1.a "...determinar los requisitos especificados } \\
\text { por el cliente". } \\
\text { 7.5.1 "...planificar y llevar a cabo la producción } \\
\text { y la prestación del servicio bajo condiciones } \\
\text { controladas". }\end{array}$ & $\begin{array}{l}\text { ISO 15489-2 4.3.2 “...decidir qué documentos se } \\
\text { incorporan...". }\end{array}$ \\
\hline $\begin{array}{c}\text { 4. Captura del conocimiento } \\
\text { Captar experiencias y lecciones } \\
\text { aprendidas }\end{array}$ & $\begin{array}{l}\text { Mantener registros de: } \\
\text { 5.6.1 revisión por la dirección; } \\
\text { 6.2.2.e educación, formación, habilidades y } \\
\text { experiencia; } \\
\text { 7.1 que los procesos de realización y el producto } \\
\text { resultante cumplen los requisitos; }\end{array}$ & $\begin{array}{l}\text { ISO } 15489-24.3 .3 \text { “...formalizar la incorporación } \\
\text { de un documento al sistema de gestión } \\
\text { documental...”. }\end{array}$ \\
\hline
\end{tabular}


María Janneth Álvarez Álvarez, Martha Castro Rojas, Guillermo Peña Guarín

\begin{tabular}{|c|c|c|}
\hline $\begin{array}{l}\text { Procesos de gestión } \\
\text { del conocimiento } \\
\text { (según UNE 412001:2008) }\end{array}$ & $\begin{array}{l}\text { Gestión de la calidad } \\
\text { (según ISO 9001:2008) }\end{array}$ & $\begin{array}{l}\text { Gestión documental } \\
\text { (según ISO 30301:2011 - } \\
\text { ISO 15489-2:2001) }\end{array}$ \\
\hline $\begin{array}{l}\text { 4. Captura del conocimiento } \\
\text { Captar experiencias y lecciones } \\
\text { aprendidas }\end{array}$ & $\begin{array}{l}\text { 7.2.2. la revisión de los requisitos del producto; } \\
\text { 7.3.2 las entradas al diseño; } \\
\text { 7.3.4 la revisión del diseño; } \\
\text { 7.3.5 la verificación del diseño; } \\
\text { 7.3.6 la validación del diseño; } \\
\text { 7.3.7 los cambios al diseño; } \\
\text { 7.4.1 la evaluación de los proveedores; } \\
\text { 7.5.3 la identificación y trazabilidad del producto; } \\
\text { 7.5.4 la propiedad del cliente; } \\
\text { 7.6 los resultados de la calibración; } \\
\text { 8.2.2 las auditorías internas; } \\
\text { 8.2.4 evidencia de la conformidad del producto; } \\
\text { 8.3 la naturaleza de las no conformidades y de } \\
\text { las acciones tomadas; } 8.5 .2 . a \text { los resultados de las } \\
\text { acciones correctivas tomadas; } \\
\text { 8.5.3 los resultados de las acciones preventivas } \\
\text { tomadas. } \\
\text { 8.2.3 “...aplicar métodos apropiados para el } \\
\text { seguimiento y, cuando sea aplicable, la medición } \\
\text { de los procesos del sistema de gestión de la } \\
\text { calidad”. }\end{array}$ & \\
\hline $\begin{array}{l}\text { 5. Adaptación del conocimiento } \\
\text { Análisis de los datos y de } \\
\text { la información para generar } \\
\text { conocimiento }\end{array}$ & $\begin{array}{l}8.4 \text { “...determinar, recopilar y analizar los datos } \\
\text { apropiados para demostrar la idoneidad y la } \\
\text { eficacia del sistema de gestión de la calidad y } \\
\text { para evaluar dónde puede realizarse la mejora } \\
\text { continua". }\end{array}$ & \\
\hline $\begin{array}{l}\text { 6. Organización del conoci- } \\
\text { miento } \\
\text { Codificación y clasificación del } \\
\text { conocimiento para facilitar su } \\
\text { acceso }\end{array}$ & $\begin{array}{l}\text { 4.2.1.d “...incorporar los documentos, incluidos } \\
\text { los registros que la organización determina } \\
\text { que son necesarios para asegurarse de la eficaz } \\
\text { planificación, operación y control de sus procesos". } \\
\text { 4.2.3 "Los documentos requeridos por el sistema } \\
\text { de gestión de la calidad deben controlarse". }\end{array}$ & $\begin{array}{l}\text { ISO } 154894.3 .4 \text { “...identificación de categorías } \\
\text { dentro de las actividades de la organización para } \\
\text { facilitar la descripción, control y acceso...". }\end{array}$ \\
\hline $\begin{array}{l}\text { 7. Almacenamiento del cono- } \\
\text { cimiento } \\
\text { Dónde se encuentra y cómo se } \\
\text { accede a él }\end{array}$ & $\begin{array}{l}\text { 4.2.4 “...establecer un procedimiento } \\
\text { documentado para definir los controles necesarios } \\
\text { para la identificación, el almacenamiento, la } \\
\text { protección, la recuperación, la retención y la } \\
\text { disposición de los registros". }\end{array}$ & $\begin{array}{l}\text { ISO 15489-2 4.3.5 “...establecimiento de derechos } \\
\text { y restricciones de acceso...”. } \\
\text { ISO 15489-2 4.3.6 “...identificar el plazo de } \\
\text { conservación del documento...”. }\end{array}$ \\
\hline
\end{tabular}




\begin{tabular}{|c|c|c|}
\hline $\begin{array}{c}\text { Procesos de gestión } \\
\text { del conocimiento } \\
\text { (según UNE 412001:2008) }\end{array}$ & $\begin{array}{l}\text { Gestión de la calidad } \\
\text { (según ISO 9001:2008) }\end{array}$ & $\begin{array}{l}\text { Gestión documental } \\
\text { (según ISO 30301:2011 - } \\
\text { ISO 15489-2:2001) }\end{array}$ \\
\hline & & $\begin{array}{l}\text { ISO } 15489-2 \text { "Los documentos que se hayan } \\
\text { retirado de los sistemas en funcionamiento } \\
\text { deberín ser accesibles y recuperables durante } \\
\text { todo su periodo de conservación". } \\
\text { ISO 15489-2 4.3.7 "...condiciones adecuadas } \\
\text { de almacenamiento garantizan la protección, la } \\
\text { accesibilidad y la buena gestión..." }\end{array}$ \\
\hline $\begin{array}{l}\text { 8. Aplicación del conocimiento } \\
\text { Entrenamiento y capacitación } \\
\text { Mejora el trabajo } \\
\text { Hay innovación }\end{array}$ & $\begin{array}{l}\text { 6.2.1 "El personal que realice trabajos que afecten } \\
\text { a la conformidad con los requisitos del producto } \\
\text { debe ser competente". } \\
8.1 \text { "...planificar e implementar los procesos } \\
\text { de seguimiento, medición, análisis y mejora } \\
\text { necesarios". } \\
\text { 8.5.1 "...mejorar continuamente la eficacia del } \\
\text { sistema de gestión de la calidad". }\end{array}$ & $\begin{array}{l}\text { ISO 15489-2 “...garantía de que todo uso de los } \\
\text { documentos se registra...”. }\end{array}$ \\
\hline
\end{tabular}

Un análisis más detallado de las relaciones entre los modelos normativos y la gestión del conocimiento muestra que el sistema de gestión de la calidad basado en ISO 9001 contiene requerimientos específicos que se pueden interpretar como generadores de cambio cultural en la organización y como facilitadores de la gestión del conocimiento, por ejemplo: la clara definición de autoridad y responsabilidad en la organización, así como también la comunicación abierta de esas disposiciones, de manera que el personal se las apropie; la promoción de la toma de conciencia en relación con los requisitos de los clientes, el impacto del trabajo de cada persona sobre la satisfacción del cliente y su contribución al logro de unos objetivos. Esto implica la toma de conciencia sobre aspectos importantes de la gestión del conocimiento, ligados a la estrategia de la organización. Además, ISO 9001 plantea la comunicación interna como un mecanismo clave para generar cultura, aspecto de la gestión del conocimiento con el que coincide plenamente.
Por otra parte, la gestión documental también incluye importantes aspectos relacionados con el cambio cultural en la organización, asociados a la gestión del conocimiento, como plantear que la organización exija a su personal documentarse correctamente, tomar consciencia de la importancia de la documentación y que en los procesos de comunicación interna se haga referencia a la gestión documental.

En cuanto al proceso de creación del conocimiento, la norma ISO 9001 contiene requisitos explícitos para su creación, al indicar en forma muy detallada cómo se debe hacer el diseño y desarrollo de nuevos productos, es decir, la innovación, en cómo aprender de los errores a través de la aplicación de acciones correctivas y en cómo aprender a evitar errores mediante el análisis de riesgos y la toma de acciones preventivas. El modelo de gestión documental no dispone de referencias que se puedan asociar a la creación de conocimiento. 
En cuanto al proceso de identificación del conocimiento, la gestión documental basada en ISO 30301 e ISO 15489-2 hace una referencia general para que se identifiquen los documentos que se deben incorporar a la gestión documental, mientras que ISO 9001 propone la necesidad de planificar el sistema de gestión de la calidad, los procesos necesarios para realizar el producto y su control. Estas actividades de planificación y control señalan hacia unas necesidades específicas de conocimiento, para que puedan ser llevadas a cabo convenientemente, y se fundamentan en un conocimiento previo acerca de reconocer los requisitos establecidos por el cliente.

En el proceso de captura del conocimiento, la gestión documental plantea únicamenten la necesidad de formalizar la incorporación de un documento al sistema de gestión documental. Por su parte, la norma ISO 9001 es altamente explícita sobre los datos y la información que se deben capturar, y se refiere al menos a dieciocho tipos de registros específicos que se deben conservar.

En cuanto a la adaptación del conocimiento, la norma ISO 9001 contiene requerimientos para que se efectúe un análisis de los datos recopilados, de manera que de este análisis surja un conocimiento que permita la mejora continua en relación con la satisfacción del cliente, los niveles de conformidad de los productos y de los servicios, las características y tendencias de los procesos y el comportamiento de los proveedores. Así, pues, del análisis de los datos se debe generar conocimiento sobre aspectos vitales para el desarrollo de la estrategia de la organización. Por su parte, la gestión documental no tiene referencias al proceso de adaptación del conocimiento.

En el proceso de organización del conocimiento, la gestión documental plantea la importancia de definir categorías dentro de las actividades de la organización, para facilitar la descripción, el control y el acceso a los documentos; este aspecto es ampliamente desarrollado en varios apartados de la guía ISO 15489-2. En cambio, la gestión de la calidad se refiere de manera general al control de los documentos y registros que la norma exige.

Para el proceso de almacenamiento del conocimiento, la norma ISO 9001 contiene un requisito general sobre el almacenamiento y conservación de los registros, mientras que la gestión documental, de acuerdo con la norma ISO 15489-2, se extiende bastante con lineamientos muy detallados que guían con mecanismos explícitos.

En relación con el proceso de aplicación del conocimiento, la gestión de la calidad basada en el modelo ISO 9001 enfatiza en la importancia de que las personas sean competentes, es decir, que conozcan y tengan la habilidad para utilizar ese conocimiento en su trabajo diario; también aborda la importancia de implementar las mejoras para incrementar la eficacia del sistema de gestión de la calidad. La gestión documental, por su parte, solo se refiere a la necesidad de evidencia del uso de los documentos. 


\section{CONCLUSIONES}

Se encuentra que la mayor convergencia entre los tres enfoques de gestión (el del conocimiento, el de la calidad y el documental) se presenta en la creación de la cultura necesaria para gestionar el conocimiento. Para fomentar esta cultura, la gestión de la calidad y la documental coinciden en la importancia de la toma de consciencia por parte del personal y en la comunicación interna, de modo que para la construcción conjunta de los tres sistemas, estos dos aspectos resultan cruciales.

Hay una gran convergencia entre el proceso de captura del conocimiento con los requerimientos puntuales que hace la gestión de la calidad para que se mantengan registros específicos, lo cual permite operacionalizar ese proceso y facilita la construcción conjunta de estos sistemas de gestión.

La gestión documental complementa muy bien a la gestión de la calidad y a la del conocimiento en lo relativo al almacenamiento de los documentos, para facilitar su conservación, disponibilidad en los plazos acordados y el acceso seguro a estos; para ello brinda orientaciones específicas al respecto.

La gestión de la calidad, tal como la define la norma ISO 9001:2008, tiene disposiciones particulares que se pueden asociar a todos los procesos de la gestión del conocimiento que propone el informe UNE 412001:2008; sin embargo, la gestión documental, definida en la norma ISO 30301:2011 y la guía ISO 15489-2:2001, no tienen ninguna referencia directa a dos de los procesos de la gestión del conocimiento: el de creación del conocimiento y el de adaptación del conocimiento.

El sistema de gestión de la calidad propone que se determinen los procesos necesarios para cumplir los compromisos con los clientes, incluyendo procesos operativos, los de soporte y los de direccionamiento; estos procesos están directamente vinculados con el quehacer diario de la organización. En cambio, la gestión del conocimiento propone unos procesos que no están en línea directa con las actividades cotidianas, sino con la propia gestión del conocimiento. Por su parte, la gestión documental es más explícita y propone que los requisitos del sistema de gestión documental se integren a los "procesos de negocio" de la organización.

Entendiendo estos procesos de negocio como los que plantea el sistema de gestión de la calidad, se deduce que el eje articulador entre la gestión del conocimiento, la de la calidad y la documental deberían ser los procesos que define el sistema de gestión de la calidad.

Llevar a cabo la gestión del conocimiento sin el soporte de la gestión documental puede resultar ineficaz, ya que esta última brinda los recursos para que los datos y la información fluyan de manera confiable a través de los procesos del negocio, y se convierte, finalmente, en la depositaria del conocimiento generado para su posterior utilización.

Los procesos de la gestión del conocimiento no tienen una secuencia lineal, sino cíclica; la aplicación del conocimiento se une con la creación del conocimiento, dando lugar a que se desarrollen de nuevo todos los demás procesos.

La importancia estratégica que tienen los datos, la información y el conocimiento se puede aprovechar mejor si la organización articula de manera consciente el sistema de gestión de la calidad (orientado a mejorar la relación con los clientes), la gestión documental (dirigida a capturar, organizar y facilitar los documentos que contienen los datos y la información) y la gestión del conocimiento (enfocada en encontrar y poner a disposición de la organización el conocimiento que requiere para mejorar su desempeño). 


\section{REFERENCIAS}

ACKOFF, Russell L. Systems thinking and thinking systems. En: System Dynamics Review. 1994, vol. 10, n. ${ }^{0} 23$, p. 175-188.

HANISCH, Bastian; LINDER, Frank; MUELLER, Ana y WALD, Andreas. Knowledge management in Project environments. En: Journal of Knowledge Management. 2009, vol. 13, n.o 4. p. 148.

CAMISÓN, César; CRUZ, Sonia y GONZÁLEZ, Tomás. Gestión de la calidad: conceptos, enfoques modelo y sistemas. Madrid: Pearson Educación, $2007.575 \mathrm{p}$.

GITLOW, Howards y GITLOW, Shelly. Cómo mejorar la calidad y la productividad con el método Deming. Bogotá: Grupo Editorial Norma, 1991. $175 \mathrm{p}$.

JURAN, J.M. y GRYNA, Frank. Análisis y planeación de la calidad. México: McGraw-Hill, 1993. 260 p.

INSTITUTO COLOMBIANO DE NORMAS TÉCNICAS Y CERTIFICACIÓN. Sistemas de gestión de la calidad. Requisitos. NTC-ISO 9001:2008. Bogotá D.C.: El Instituto, 2009, 35 p.

INSTITUTO COLOMBIANO DE NORMAS TÉCNICAS Y CERTIFICACIÓN. Sistemas de gestión de la calidad. Fundamentos y vocabulario. NTC-ISO 9000:2005. Bogotá D.C.: El instituto, 2005, $36 \mathrm{p}$.
ARTILES, Sara. La gestión documental, de información y el conocimiento en la empresa. En: ACIMED. 2009, vol. 19, n.o 5, 37 p.

INSTITUTO COLOMBIANO DE NORMAS TÉCNICAS Y CERTIFICACIÓN. Directrices para la documentación del sistema de gestión de la calidad. GTC-ISO/TR 10013. Bogotá D. C.: El Instituto, 2002. $16 \mathrm{p}$.

INTERNATIONAL ORGANIZATION FOR STANDARDIZATION. Information and Documentation - Records Management - Part 1. General. ISO 15489-1:2001 Geneva: Same, 2001. 12 p.

INTERNATIONAL ORGANIZATION FOR STANDARDIZATION. Information and Documentation - Records Management - Part 2. Guidelines. ISO 15489-1:2001 Geneva: Same, 2001. $23 \mathrm{p}$.

INTERNATIONAL ORGANIZATION FOR STANDARDIZATION. Information and Documentation - Management System for records. ISO 30301:2011 Geneva: Same, 2011. 22 p.

INTERNATIONAL ORGANIZATION FOR STANDARDIZATION. Guidelines for quality management system documentation. ISO/TR 10013:2001.Geneva. 2001. 14 p.

ASOCIACIÓN ESPAÑOLA DE NORMALIZACIÓN Y GERTIFICACIÓN. Guía práctica de gestión del conocimiento. UNE 412001:2008. Madrid: La Asociación, 2008. 117 p. 